\title{
A TECHNIQUE FOR CHARACTERIZING CARLESON MEASURES ON BERGMAN SPACES
}

\author{
DANIEL LUECKING
}

\begin{abstract}
A method is presented for characterizing Carleson-type measures relative to Bergman spaces. This method applies to the standard weighted and unweighted Bergman spaces on the unit ball in $\mathbf{C}^{n}$ to yield simple proofs of the known results. It also extends these results to domains more general than balls.
\end{abstract}

Let $D$ be a domain in $\mathbf{C}^{n}$ and $A$ a space of analytic functions with a norm $\|f\|$ defined by some integral or integrals of $|f|^{p}$ being bounded. For example if $D$ is the unit disk in $C^{1}, A$ could be an $H^{p}$ space or a Bergman space. If $\mu$ is a positive measure on $D$, a common terminology is to call $\mu$ an $A$-Carleson measure if there is a constant $C>0$ such that

$$
\left(\int_{D}|f|^{p} d \mu\right)^{1 / p} \leqslant C\|f\| .
$$

This note will deal only with Bergman spaces $A^{p}(w, D)$ defined for $p>0$ and nonnegative $w$ by $A^{p}(w, D)=\left\{f: f\right.$ is analytic in $D$ and $\left.\int_{D}|f|^{p} w d m<+\infty\right\}$. The integral is with respect to Lebesgue $2 n$-dimensional volume measure (or area if $n=1)$. Associate with each $z \in D$ an open set $E(z)$ containing $z$ with the following properties:

(1) $E(z) \subseteq D$ and $\chi_{E(z)}(\zeta)$ is measurable in $D \times D$.

(2) There is a constant $C_{1}>0$ such that

$$
|\bigcup\{E(z): E(z) \cap E(a) \neq \varnothing\}|<C_{1}|E(a)| \text {. }
$$

(3) There is a constant $C_{2}>0$ such that for all $a \in D, w\left(z_{1}\right)<C_{2} w\left(z_{2}\right)$ when $z_{1}$, $z_{2} \in E(a)$.

$(|\cdot|$ denotes the Lebesgue measure of a set.)

EXAMPLE. Let $D$ be the unit disk in $C$ and let $r>0$. Let $E(z)$ be the hyperbolic disk about $z$ with hyperbolic radius $r$. If $w(z)=(1-|z|)^{\alpha}$ for $\alpha>-1$ then (1)-(3) are satisfied.

Let $E^{2}(y)=\bigcup\{E(z): E(y) \cap E(z) \neq \varnothing\}$.

LEMma 1. Suppose there is a constant $C>0$ such that

$$
|f(z)|^{p} \leqslant \frac{C}{|E(z)|} \int_{E(z)}|f|^{p} d m, \quad f \in A^{p}(w, D),
$$

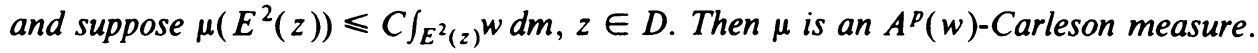

Received by the editors October 23, 1981 and, in revised form, June 16, 1982. 1980 Mathematics Subject Classification. Primary 30D60, 32A30, 31B05, 46E15.

'Supported by NSF Grant MCS-8201603.

(C) 983 American Mathematical Society 0002-9939/82/0000-0827/\$02.00 
Proof.

$$
\begin{aligned}
\int_{D}|f(z)|^{p} d \mu & \leqslant C \int_{D}\left[\frac{1}{|E(z)|} \int_{D} \chi_{E(z)}(y)|f(y)|^{p} d m(y)\right] d \mu(z) \\
& \leqslant C \int_{D}|f(y)|^{p}\left[\int_{D} \frac{1}{|E(z)|} \chi_{E(z)}(y) d \mu(z)\right] d m(y) \\
& \leqslant C \int_{D}|f(y)|^{p} \int_{D} \frac{1}{|E(z)|} \chi_{E^{2}(y)}(z) d \mu(z) d m(y) .
\end{aligned}
$$

ReMARK. The obvious inequality $\chi_{E(z)}(y) \leqslant \chi_{E^{2}(y)}(z)$ has been used. (Note the interchange of position of $y$ and $z$.) Now

$$
\begin{aligned}
\int_{D}|f(z)|^{p} d \mu & \leqslant C C_{1}^{2} \int_{D}|f(y)|^{p} \frac{\mu\left(E^{2}(y)\right)}{|E(y)|} d m(y) \\
& \leqslant C^{2} C_{1}^{2} \int_{D}|f(y)|^{p} \frac{1}{|E(y)|} \int_{E^{2}(y)} w(z) d m(z) d m(y) \\
& \leqslant C^{2} C_{1}^{2} C_{2}^{2} \int_{D}|f(y)|^{p} w(y)\left|\frac{E^{2}(y)}{E(y)}\right| d m(y) \\
& \leqslant C^{2} C_{1}^{3} C_{2}^{2} \int_{D} \mid f(y) P^{p} w(y) d m(y) .
\end{aligned}
$$

Note that if $z \in E^{2}(y)$ then $z \in E(x)$ where $E(x)$ meets $E(y)$. Then (2) implies $|E(y)| \leqslant\left|E^{2}(x)\right| \leqslant C_{1}|E(x)| \leqslant C_{1}\left|E^{2}(z)\right| \leqslant C_{1}^{2}|E(z)|$. Thus $1 /|E(z)| \leqslant$ $C_{1}^{2} /|E(y)|$. This is how (4) comes about.

Before applying this lemma I will mention some previous work. Carleson measures for $H^{p}$ spaces were introduced by Carleson $[\mathbf{C}]$. The unweighted Bergman spaces in the disk and polydisk $\left(A^{p}(w)\right.$ with $\left.w=1\right)$ were studied by Hastings, and $A^{p_{-}}$ Carleson measures were characterized in $[\mathbf{H}]$. Stegenga in [S] characterized $A^{2}\left((1-|z|)^{-1-2 \alpha}, D\right)$-Carleson measures with $D$ the unit disk and $\alpha<0$. The case where $D$ is the unit ball in $\mathbf{C}^{n}$ and $w(z)=\left(1-|z|^{2}\right)^{\beta}, \beta>-1$, was obtained by Cima and Wogen [CW].

The technique for characterizing Carleson measures on Bergman spaces is the application of Lemma 1 with appropriate choices of $E(z)$ and $w(z)$. This technique is very similar to one developed by V. L. Oleinik in [O] but it avoids a technical covering lemma that Oleinik employs. My substitute seems to be the application of Fubini's Theorem in Lemma 1. Moreover, no special assumptions need be made about the regularity of $E(z)$ as $z$ varies nor are any boundness assumptions on $1 / w$ needed. Moreover, Oleinik limits $E(z)$ to be balls.

Lemma 1 can be used to supply sufficient conditions that $\mu$ be an $A^{p}(w)$-Carleson measure. To show that these conditions are necessary usually involves ad hoc construction of functions in $A^{P}(w)$.

I illustrate the application of Lemma 1 with two examples. The first is a proof of Cima and Wogen's result on the ball. Let $B$ denote the unit ball in $\mathbf{C}^{n}$. For $z \in B$ let $\phi_{z}$ be any one-to-one holomorphic map of $B$ onto $B$ that interchanges 0 and $z$. See Rudin [R] for the following properties of $E(z)$ and $\phi_{z}$. Let $E(0)$ be a ball about 0 
with radius $\frac{1}{2}$ and let $E(z)=\phi_{z}(E(0)) . E(z)$ is an ellipsoid which cuts the complex line $\mathbf{C} \cdot \boldsymbol{z}$ in a disk with radius proportional to $\mathbf{l}-|z|$. It cuts complex lines through $z$ perpendicular to $\mathbf{C} \cdot z$ in disks with radius proportional to $\sqrt{1-|z|}$. Thus $|E(z)|$ is proportional to $(1-|z|)^{n+1}$.

For $\eta \in \partial B$ and $\tau>0$ let

$$
S(\eta, \tau)=\{z \in B:|1-\langle z, \eta\rangle|<\tau\}
$$

where $\langle\cdot, \cdot\rangle$ is the complex inner product on $\mathbf{C}^{n} . S(\eta, t)$ has dimensions similar to $E(z)$. That is, in $\mathbf{C} \eta$ it is the intersection of the unit disk with a disk centered at $\eta$ and radius $t$, while in perpendicular directions it has dimensions on the order of $\sqrt{t}$. Given $z \in B$ and $\eta=z /|z|$, choose $t>0$ to be the smallest number such that $E(z) \subseteq S(\eta, t)$. Then $t$ is proportional to $1-|z|$ and $|E(z)|$ is proportional to $|S(\eta, t)|$. Moreover, if $v$ is the measure $(1-|z|)^{\beta} d m(z), \beta>-1$, then $v(E(z)) \sim$ $v(S(\eta, t)$ ). (The word "proportional" and the symbol " " indicate the two quantities have a ratio that is bounded and bounded away from zero as $z$ varies.)

Proposition 1 (Cima ANd Wogen). A positive measure $\mu$ on $B$ is an $A^{p}(v, B)$ Carleson measure if and only if there is a constant $C>0$ such that

$$
\mu(S(\eta, t)) \leqslant C v(S(\eta, t)), \quad \eta \in \partial B, t>0 .
$$

REMARK. Cima and Wogen take (5) as their definition of Carleson measure. Their proof uses certain maximal functions and Marcinkiewicz interpolation and is patterned after the proof in Duren [D] of the $H^{p}$ result. The present proof seems more suited to the situation of an area integral.

Proof. From the previous discussion, and obvious geometry it is clear that (5) implies there is a $C>0$ such that

$$
\mu\left(E^{2}(z)\right) \leqslant C v\left(E^{2}(z)\right), \quad z \in B .
$$

[For $E^{2}(z)=\phi_{z}\left(E^{2}(0)\right)$ and $E^{2}(0)$ is a ball about zero of some radius $r_{0}, \frac{1}{2}<r_{0}<1$, so the above discussion applies.] Now

$$
\left|f\left(\phi_{z}(0)\right)\right|^{p} \leqslant \frac{1}{|E(0)|} \int_{E(0)}\left|f\left(\phi_{z}(\zeta)\right)\right|^{p} d m(\zeta)
$$

and a change of variables in the integral $\left(\zeta=\phi_{z}^{-1}(\xi)\right)$ gives

$$
|f(z)|^{p} \leqslant \frac{1}{|E(0)|} \int_{E(z)}|f(\xi)|^{p}\left|\operatorname{det} \phi_{z}^{\prime}(\zeta)\right|^{-2} d m(\xi) .
$$

Now

$$
\left|\operatorname{det} \phi_{z}^{\prime}(\zeta)\right|^{2}=\left(\frac{1-|z|^{2}}{|1-\langle\zeta, z\rangle|^{2}}\right)^{n+1} \sim|E(z)| .
$$

Note that $\zeta \in E(0)$ implies the denominator is bounded away from zero. Thus

$$
|f(z)|^{p} \leqslant C \frac{1}{|E(z)|} \int_{E(z)}|f|^{p} d m \text { for some } C .
$$

The hypotheses of Lemma 1 are therefore satisfied and one implication of the proposition is proved provided (1)-(3) are verified. But these are easy consequences 
of the definition of $E(z)$ and the properties discussed prior to the proposition. The proof of the converse implication given by Cima and Wogen is quite standard and cannot be improved. It amounts to estimating the integral of an appropriate power of $|1-\langle z, \zeta\rangle|$ for $\zeta=(1-t) \eta$, using for example the estimates in Rudin [R, Proposition 14.10]. Q.E.D.

A close inspection of the above proof shows it is based on the following properties of $B$ : (a) $B$ is homogeneous and (b) the Bergman kernel $K(z, \zeta)$ satisfies $K(\zeta, \zeta)<$ $C K(z, z)$ when $\zeta \in E(z)$. (This is because

$$
\left|\operatorname{det} \phi_{z}^{\prime}(\zeta)\right|^{2}=\frac{K(\zeta, \zeta)}{K\left(\phi_{z}(\zeta), \phi_{z}(\zeta)\right)}<\frac{C^{2} K(0,0)}{K(z, z)}, \quad \zeta \in E(0),
$$

and $K(z, z) \sim 1 /|E(z)|$.) Thus a result analogous to the direction proved here of Proposition 1 is valid in domains satisfying (a) and (b) provided $S(\eta, t)$ is replaced by $E(z)$. Examples of domains with these properties are symmetric Siegel domains of type two, studied by Coifman and Rochberg in $[\mathbf{C R}]$. In that case $E(z)$ are balls in the Bergman metric of constant radius.

The second application is even simpler. It has the advantage of being completely general in application but the disadvantage of not having a converse. Let $D$ be any domain in $C^{n}$. For $0<\tau<1$ and $z \in D$, let $B_{\tau}(z)=\{\zeta \in D:|\zeta-z|<$ $\tau \cdot \operatorname{dist}(z, \partial D)\}$ so that the $B_{\tau}(z)$ are balls centered at $z$. It is clear that $B_{\tau}(z)$ satisfy conditions (1) and (2) on $E(z)$.

Proposition. Suppose there is a constant $C_{1}>0$ such that $w(\zeta)<C_{1} w(z)$ whenever $\zeta \in B_{\tau}(z)$, and suppose $\mu$ is a positive measure on $D$ such that

$$
\mu\left(B_{\tau}(z)\right) \leqslant C_{2} \int_{B_{\tau(z)}} w d m, \quad z \in D,
$$

then there exists a constant $C>0$ such that $\int_{D} g d \mu \leqslant C \int_{D} g w d m$ for all nonnegative subharmonic functions $g$.

Proof. $B_{\tau}(z)$ and $w$ satisfy (1)-(3) and $g$ satisfies the condition on $|f|^{p}$ in Lemma 1. Thus the proposition will be proved if it can be shown that

$$
\mu\left(B_{\tau}^{2}(z)\right) \leqslant C \int_{B_{\tau}^{2}(z)} w d m, \quad z \in D,
$$

where $B_{\tau}^{2}(z)=\cup\left\{B_{\tau}(\zeta): B_{\tau}(\zeta) \cap B_{\tau}(z) \neq \varnothing\right\}$. This is simple geometry: There is a constant $M$ such that $B^{2}(z)$ can be covered by at most $M$ balls $B_{\tau}\left(z_{k}\right), z_{k} \in$ $B_{\tau}^{2}(z)$.Then

$$
\begin{aligned}
\mu\left(B_{\tau}^{2}(z)\right) & \leqslant \sum_{k=1}^{M} \mu\left(B_{\tau}\left(z_{k}\right)\right) \leqslant C_{2} \sum_{k=1}^{M} \int_{B_{\tau}\left(z_{k}\right)} w d m \\
& \leqslant C \sum_{k=1}^{M} w\left(z_{k}\right)\left|B_{\tau}\left(z_{k}\right)\right| \leqslant C M w(z)\left|B_{\tau}\left(z_{k}\right)\right| \\
& \leqslant C \int_{B_{\tau}(z)} w d m \leqslant C \int_{B_{\tau}^{2}(z)} w d m .
\end{aligned}
$$


Note. Not all $C$ 's have the same value and the fact $w(\zeta)<C_{1} w(z)$ for $\zeta \in B_{\tau}(z)$ has been used several times. Q.E.D.

An alternate proof, not appealing to any covering, can be obtained by repeating the argument of Lemma $l$ with $g$ in place of $|f|^{p}$ until

$$
\int_{D} \frac{\chi_{B_{\tau}(z)}(y)}{\left|B_{\tau}(z)\right|} d \mu(z)
$$

must be estimated (after the second $\leqslant$ ). Then use

$$
\chi_{B_{\tau}(z)}(y) \leqslant \frac{C}{\left|B_{\tau}(z)\right|} \int_{B} \chi_{B_{\tau}(y)}(\zeta) \chi_{B_{\tau}(\zeta)}(z) d m(\zeta) .
$$

Using $B(z)$ as abbreviation for $B_{\tau}(z)$ and applying Fubini's Theorem gives

$$
\begin{aligned}
\int_{D} \frac{\chi_{B(z)}(y)}{|B(z)|} d \mu(z) & \leqslant C \int_{D} \chi_{B(y)}(\zeta) \int_{D} \frac{\chi_{B(\zeta)}(z)}{|B(z)|^{2}} d \mu(z) d m(\zeta) \\
& \leqslant C \int_{D} \chi_{B(y)}(\zeta) \frac{\int_{B(\zeta)} d \mu}{|B(\zeta)|^{2}} d m(\zeta) \\
& \leqslant C \frac{\int_{D} \chi_{B(y)}(\zeta) w(\zeta) d m(\zeta)}{|B(y)|} \leqslant C w(y)
\end{aligned}
$$

When this is inserted in

$$
\int g d \mu \leqslant C \int_{D} g(y) \int_{D} \frac{\chi_{B(z)}(y)}{|B(z)|} d \mu(z) d m(y),
$$

we get $\int g d \mu \leqslant C \int g w d m$ as required.

\section{REFERENCES}

[C] L. Carleson, Interpolation by bounded analytic functions and the corona problem, Ann. of Math. (2) 76 (1962), 547-559.

[CW] J. A. Cima and W. R. Wogen, A Carleson measure theorem for the Bergman space on the ball, preprint.

[CR] R. R. Coifman and R. Rochberg, Representation theorems for holomorphic and harmonic functions, Astérisque 77 (1980), 11-65.

[D] P. L. Duren, Theory of $H^{p}$ spaces, Academic Press, New York, 1970

[H] W. W. Hastings, A Carleson measure theorem for Bergman spaces, Proc. Amer. Math. Soc. 52 (1975), 237-241.

[O] V. L. Oleinik, Embedding theorems for weighted classes of harmonic and analytic functions, J. Soviet Math. 9 (1978), 228-243.

[R] W. Rudin, Function theory in the unit ball in $\mathbf{C}^{n}$, Springer-Verlag, Berlin and New York, 1980.

[S] D. A. Stegenga, Multipliers of the Dirichlet space, Illinois J. Math. 24 (1980), 113-139.

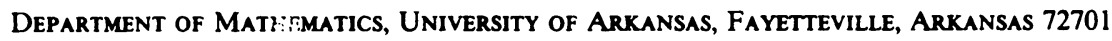

